\title{
The application of a shift theorem analysis technique to multipoint measurements
}

\author{
M. E. Dieckmann, S. C. Chapman \\ Space and Astrophysics Group, University of Warwick, Coventry, CV 47 AL, UK \\ E-mail: Dieckmann@astro.warwick.ac.uk \\ Received: 17 February 1997 / Revised: 19 August 1998 / Accepted: 3 September 1998
}

\begin{abstract}
A Fourier domain technique has been proposed previously which, in principle, quantifies the extent to which multipoint in-situ measurements can identify whether or not an observed structure is time stationary in its rest frame. Once a structure, sampled for example by four spacecraft, is shown to be quasistationary in its rest frame, the structure's velocity vector can be determined with respect to the sampling spacecraft. We investigate the properties of this technique, which we will refer to as a stationarity test, by applying it to two point measurements of a simulated boundary layer. The boundary layer was evolved using a PIC (particle in cell) electromagnetic code. Initial and boundary conditions were chosen such, that two cases could be considered, i.e. a spacecraft pair moving through (1) a time stationary boundary structure and (2) a boundary structure which is evolving (expanding) in time. The code also introduces noise in the simulated data time series which is uncorrelated between the two spacecraft. We demonstrate that, provided that the time series is Hanning windowed, the test is effective in determining the relative velocity between the boundary layer and spacecraft and in determining the range of frequencies over which the data can be treated as time stationary or time evolving. This work presents a first step towards understanding the effectiveness of this technique, as required in order for it to be applied to multispacecraft data.
\end{abstract}

Key words. Electromagnetics (wave propagation), Radio science (waves in plasma), Space plasma physics (active perturbation experiments).

\section{Introduction}

An unambiguous understanding of the structure and dynamics of the Earth's environment requires multi-

Correspondence to: M. E. Dieckmann point measurements to distinguish between spatial and temporal changes in the field and plasma. Techniques are then needed to combine the in-situ data from more than one spacecraft to quantify to what extent the observed structure can be treated as time stationary, with a single relative velocity with respect to the spacecraft, or time evolving in the structure's rest frame. We will refer to the test determining the time stationarity as a stationarity test. A simple stationarity test was proposed by Chapman and Dunlop (1993) and will be investigated here. This is complementary to approaches, where a specific quantity (such as $\nabla \wedge \vec{B}$ ) (Dunlop et al., 1988, Dunlop, 1990, Dunlop et al., 1990) or a sample of the spectrum (Neubauer et al., 1990) are deduced from the multispacecraft data. For further work on multipoint data analysis see also (CLUSTER Workshop, 1994). We demonstrate the effectiveness of this stationarity test (Chapman and Dunlop, 1993) by means of simulated multispacecraft data. The data is obtained by flying 'virtual' spacecraft' i.e. sampling the time evolving solution of a simple boundary layer. The boundary layer solution is generated by the $1 \frac{1}{2} \mathrm{D}$ (one spatial and three velocity components are solved for) fully electromagnetic particle in cell code described in Devine et al., (1995). Initial and boundary conditions were chosen to generate (1) a time stationary boundary and (2) a time evolving boundary. The simulation also naturally provides uncorrelated noise, so that we are able to examine the effectiveness of the technique in the presence of noise in the simulated data. Results for two point measurements are presented, but the technique can easily be generalized to more than two points.

\section{The stationarity test}

The stationarity test considered, described in Chapman and Dunlop (1993), makes use of the shift theorem to combine the amplitude and phase spectra of two datasets. A structure that is time stationary in its rest frame, and which is sampled by two spacecraft during a common sampling interval $T$, must fulfil two conditions: 
$\frac{A_{i}(f)}{A_{i+1}(f)}=1$

$\Phi_{i}(f)-\Phi_{i+1}(f)=2 \pi \delta t f$

where $A_{i}$ denotes the amplitude spectrum and $\Phi_{i}$ the phase spectrum of the dataset sampled by the $i$ th spacecraft. If the structure is coincident with the $i$ th spacecraft at time $t$, it is coincident with the $(i+1)$ th spacecraft at time $t+\delta t$. More generally, the Fourier transform will have frequency regions which obey Eqs. (1) and (2) and regions which do not. If the frequency band associated with time evolution is sufficiently well separated from the rest of the power spectrum Eqs. (1) and (2) can still effectively determine the speed of any underlying time stationary structure's frame of reference with respect to the spacecraft. In addition, noise will perturb the amplitude ratio and phase delay even though the structure itself may be stationary. However, for noise at realistic levels compared with the signal on the frequency range of interest, one can still distinguish stationary from non-stationary frequency regions as we shall see.

Since, in general, the sampled datasets are not periodic, the Fourier transform will mix the amplitude and phase information of the 'real' structure, that means the sampled physical object, with that of the discontinuity at the dataset's boundaries (a discrete Fourier transform assumes a periodic continuation of the data set). This aliasing of information can have strong effects on the test's performance as we shall see later.

By using time domain windows, the characteristics of this aliasing process can be influenced (Hamming 1993). Multiplying a time domain window with a weight of zero at both ends of the boundary will remove the unphysical discontinuity at the data interval boundary but it will also influence the 'physical' data. Two effects on the frequency distribution have to be expected. The window will smooth the frequency spectrum and it will emphasize events which are located at positions in the dataset with a strong time domain window weight. As we will see, for the case of a boundary crossing, the aliasing mentioned poses more serious problems to the test than the error introduced by the window.

Throughout this work unless stated otherwise, we used a Hanning time domain window. The windowing operation is:

$M(t)=S(t) \cdot W(t)$

where $S$ is the signal, $W$ the window and $M$ the modified signal. The dot corresponds to a multiplication operation.

The Hanning window is given by:

$W(t)=\frac{1}{2}\left(1+\cos \left(2 \pi \frac{t}{\bar{T}}-\pi\right)\right)$

with $\bar{T}$ the window width. The window coefficient is zero at and outside the interval limits 0 and $\bar{T}$. In what follows, the window width $\bar{T}$ will be set to the sampling interval $\overline{\mathrm{T}}$ which means no loss of generalization.
After locating the frequency ranges for which the structure is time stationary, as indicated by the present test, the signal in these ranges can be separated off by a frequency domain window. This will be demonstrated here, when we subdivide the spectrum into time stationary and time evolving regions by using rectangular low and bandpass filters and apply a backwards Fourier transform to them. The obtained timeseries have the properties predicted by the test, i.e. we can identify them either as stationary or as time evolving. This will act as a confirmation of the test's result.

\section{Description of the analyzed datasets}

\subsection{Initial conditions and dimensions}

Two datasets generated by a $1 \frac{1}{2} \mathrm{D}$ electromagnetic PIC (particle in cell) code with periodic boundary conditions (Devine et al., 1995) were analyzed. As structure to investigate we chose a simple boundary layer connecting a region with high magnetic pressure and low particle pressure to a region with no magnetic pressure and high particle pressure. A magnetic field component perpendicular to the simulation direction, that is the spatial coordinate solved for by the code, provided the magnetic pressure. One run had no magnetic field component parallel to the simulation direction which resulted in a quasi-stationary boundary. In the second, non-stationary run, a magnetic field component parallel to the simulation direction was introduced allowing particles to cross, and waves to propagate away from the boundary layer. The electrons in the low particle pressure region were represented by 16 particles per cell, in the high particle pressure region by 32 particles per cell. Protons were represented by a fixed and continuous charge background and the excess charge of the high particle pressure box by 16 (mobile) protons per cell. These protons, however, can be considered at rest for the simulation time scales and were introduced simply to ensure charge neutrality. The subdivision of the simulation box into two halves with different particle pressures and perpendicular magnetic fields was done as follows: Grid cells 1-4096 will be denoted as box 1 and grid cells $4097-8192$ as box 2 . The grid cell length $\Delta x$ was set to the Debye length of the plasma in box $1(1.5$ gyroradii, $8000 \mathrm{~m})$. All positions will henceforth be given as $\tilde{x}=x / \Delta x$.

The physical parameters were:

1. Electron parameters:

Number density (box 1): $6.2 \cdot 10^{4} \mathrm{~m}^{-3}$, (box 2): $3.1 \cdot 10^{4} \mathrm{~m}^{-3}$

Temperature (box 1): $4.2 \cdot 10^{8} \mathrm{~K}$, (box 2): $4.2 \cdot 10^{8} \mathrm{~K}$ Represented by (box 1): 32 particles per cell, (box 2): 16 particles per cell.

2. 'Mobile' proton parameters:

number density (box 1): $3.1 \cdot 10^{4} \mathrm{~m}^{-3}$

'Temperature (box 1): $4.2 \cdot 10^{8} \mathrm{~K}$

Represented by (box 1): 16 particles per cell.

3. Fixed proton parameters: 
Number density (box 1): $3.1 \cdot 10^{4} \mathrm{~m}^{-3}$, (box 2): $3.1 \cdot 10^{4} \mathrm{~m}^{-3}$

Continuous, fixed charge background introduced by setting the total box charge to zero.

4. Perpendicular magnetic $B_{\perp}$ field:

Field strength (box 1): zero, (box 2): $30 \mathrm{nT}$

5. Parallel magnetic $B_{\|}$field:

Zero for the quasi stationary run, $170 \mathrm{nT}$ for the time evolving run.

The magnetic pressure in box 2 is $P_{\text {mag }}=3.6 \cdot 10^{-10} \mathrm{Nm}^{-2}$ which is the same as the excess particle pressure in box 1. Therefore, the boundary evolves to a quasi equilibrium.

One simulation time step was $\Delta t=9.4 \cdot 10^{-6} \mathrm{~s}$. Thus, one electron gyroperiod for the $B_{\perp}$ field in box 2 was equivalent to $126 \Delta t$. The simulation 'band width' (range of frequency and wave number) is not sufficiently high to resolve the high frequency and low wave number modes (ordinary mode, Bernstein modes). Since the time evolution that we observe in the simulation is well described by Whistler mode propagation plus incoherent noise, we can assume that these low wave number modes are relatively weak and do not contribute to the coherent structure of interest.

All times will be given as $\tilde{t}=t / \Delta t$ with $t$ denoting the physical time and $\tilde{t}$ the simulation time. The initial particle density distribution was a step function with jumps at $\tilde{x}=4096$ (box centre) and $\tilde{x}=8192$ (box end). The magnetic field change was modelled using a $\tan \mathrm{h}$ function for the two boundaries at $\tilde{x}=4096,8192$; the boundary width was $12 \Delta x$ (18 gyroradii). A smooth change in the magnetic field was necessary to ensure the resolution of the boundary behaviour.

\subsection{Description of the simulation}

We investigated the datasets given by the two simulations. The virtual spacecraft, providing the two spacecraft data, started at two different simulation grid cells and sampled consecutively the perpendicular magnetic field. The grid cell number to be sampled was increased by one every time step, which means that the spacecraft velocity was $\Delta x / \Delta t$ directed towards higher grid cell numbers.

The datasets obtained will contain three components: The 'physical' boundary, the data interval boundary (i.e. the first and the last values of the finite length dataset) and uncorrelated noise.

We will investigate the behaviour of the test for a time evolving and a time stationary 'physical' boundary. The time stationary dataset will address the information aliasing introduced by the data interval boundary due to a non periodicity of the dataset. The time evolution of the second simulation demonstrates, how non stationarity would show up in the test's results.

The uncorrelated noise will be present in both simulations and we shall see that the test is able to distinguish between signal dominated and noise dominated frequency components. Noise is introduced due to the finite computational particle number density and the resulting statistical phase space distribution fluctuations. The fluctuating currents and density induce fluctuating fields.

For both simulations, the total run time of $27900 \Delta t$ (220 electron gyroperiods) was subdivided into two intervals. The first interval, from $\tilde{t}=0$ to $\tilde{t}=20000(158$ electron gyroperiods) was to relax the system to an equilibrium for the electrons. The boundary width increased its value to $20 \Delta x$ (30 electron gyroradii) when $\tilde{t}=1000$ and to $24 \Delta x$ (36 electron gyroradii) at $\tilde{t}=27900$, the end of the simulation (for the time stationary run). The second interval, the sampling interval, started at $\tilde{t}=20001$ and ended at $\tilde{t}=27900$, the simulation end. (62 electron gyroperiods)

3.2.1 The time stationary run. A stack plot of the $B_{\perp}$ field for the time stationary simulation is shown by Fig. 1 . The field is sampled every $650 \Delta t$ starting at $\tilde{t}=20800$ and thus shows the time evolution of the boundary during the sampling time. There is no time evolution visible. The positions of the sampling spacecraft at the times the magnetic field inside the simulation box was recorded are denoted by a $(+)$ for spacecraft 1 and a(*) for spacecraft 2. For both simulations, this and the simulation of a time evolving boundary, spacecraft 1 started at $\tilde{t}=20001$ at position $\tilde{x}=1$, spacecraft 2 at the time at $\tilde{x}=201$ which is thus the time delay in units of the simulation time step. As already mentioned, their velocity was $\Delta x / \Delta t$, directed towards higher $\tilde{x}$. At $\tilde{t}=27900$, the simulation end, spacecraft 1 reached $\tilde{x}=7900$ and spacecraft $2 \tilde{x}=8100$ for both simulations.

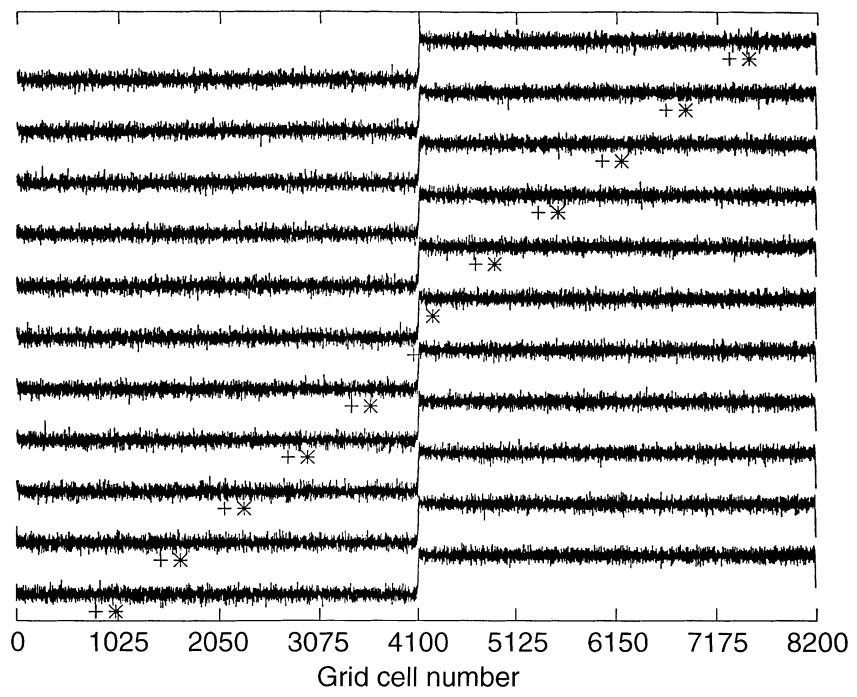

Fig. 1. Stack plot of the magnetic $B_{\perp}$ components for the sampling interval and for the time stationary run. The symbol $(+)$ denotes the position $\tilde{x}$ of spacecraft 1 and a (*) that of spacecraft 2 for the simulation time step $t$ at which the magnetic field was plotted. The exact vertical position of these position indicators is not significant; they are plotted just below the $B_{\perp}$ curve to which they refer. The abscissa shows $\tilde{x}$ 
3.2.2 The time evolving run. Time evolution was initiated by switching on a magnetic field $B_{\|}=170 \mathrm{nT}$ at $\tilde{t}=23700$. A non zero $B_{\|}$components allows the simulation particles to cross the boundary and the $B_{\perp}$ component to propagate in the whistler mode. A stack plot of the time evolving run is shown by Fig. 2. As in Fig. 1, the stack plots shows the magnetic field inside the simulation box during the time the samples were made. Again, a (+) shows the position of spacecraft (1) and a (*) that of spacecraft 2 for the time step when the field was recorded. The $B_{\perp}$ field is again sampled every 650 $\Delta t(\approx 5.1$ cyclotron periods) starting at $\tilde{t}=20800$. The onset of the time variation can be seen at sample 6 equivalent to $\tilde{t}=24050$. The noise has the same amplitude as in Fig. 1 and we will get the same type of nonperiodicity into our two datasets. By comparing the test results for the time evolving and time stationary datasets, we can investigate how efficiently they can be distinguished.

\section{Application of the stationarity test}

\subsection{Simulation data, time stationary boundary}

We now examine the stationarity test process on the data sampled by the two virtual spacecraft for the two simulations.

Figure 3 shows the $B_{\perp}$ field data as sampled by the spacecraft between $\tilde{t}=23400$ and $\tilde{t}=24600$.

The power spectra of the unwindowed spacecraft 1 data (upper plot) and of the Hanning windowed spacecraft 1 data (lower plot) are shown by Fig. 4 . The plots cover, like all frequency domain plots throughout this discussion, one half of the Fourier

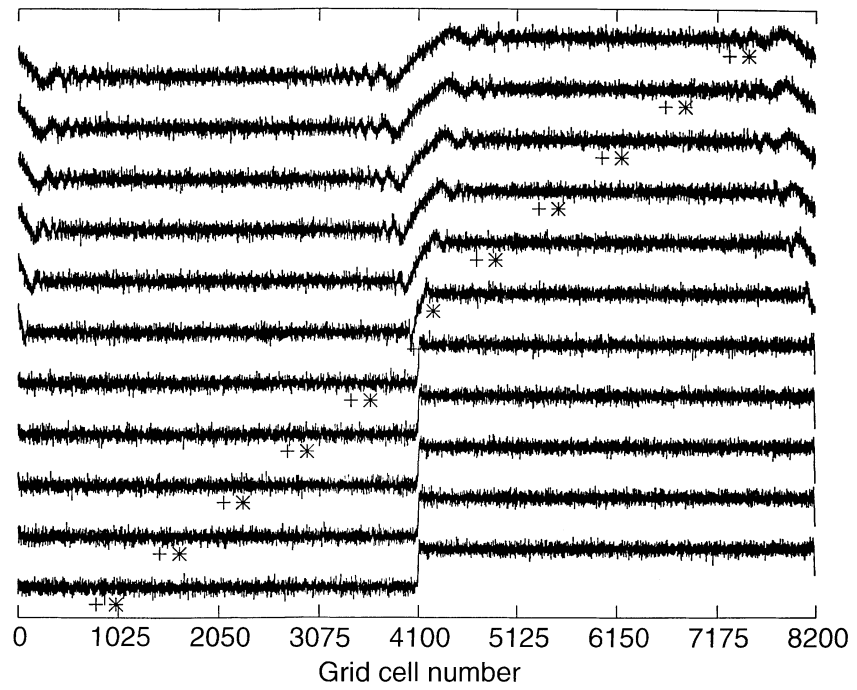

Fig. 2. Stack plot of the magnetic $B_{\perp}$ components for the sampling time. The abscissa denotes the position $\tilde{x}$ in the box. This stack plot corresponds to the time evolving run. $(+)$ denotes the position of spacecraft 1 and $(*)$ that of spacecraft 2 for the simulation time step $\tilde{t}$ at which the magnetic field was plotted. For the position indicators, the same holds true as in Fig 1 spectrum up to the Nyquist frequency excluding the DC offset. The frequencies are expressed in units of $\Delta f=1 / T$ with the sampling time $T=63$ electron gyroperiods.

The fluctuations occurring at frequencies up to $100 \Delta f$ in the upper plot result from mixing of the amplitude and phase of the 'physical' boundary and the discontinuous data interval boundary. They have been removed by the multiplication of the Hanning window to the time domain data before Fourier transforming them.

Figure 5 shows the stationarity test's result for the unwindowed datasets. The abscissas correspond to the
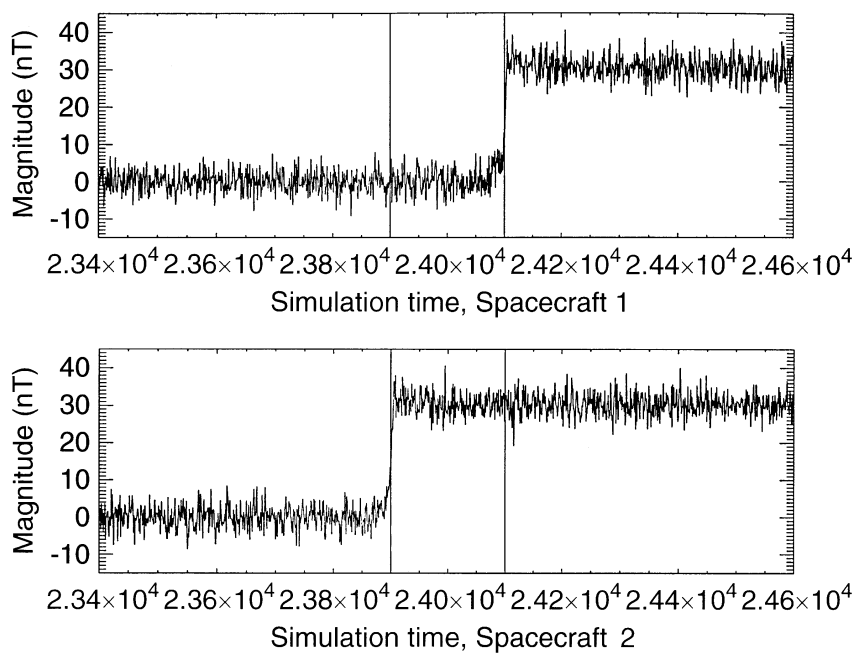

Fig. 3. The magnetic $B_{\perp}$ field component as sampled by the spacecraft for the time stationary run. The abscissa shows the simulation time $\tilde{t}$ and the ordinate the corresponding magnetic field. The samples of higher and lower sampling time steps are clipped, since they only represent noise
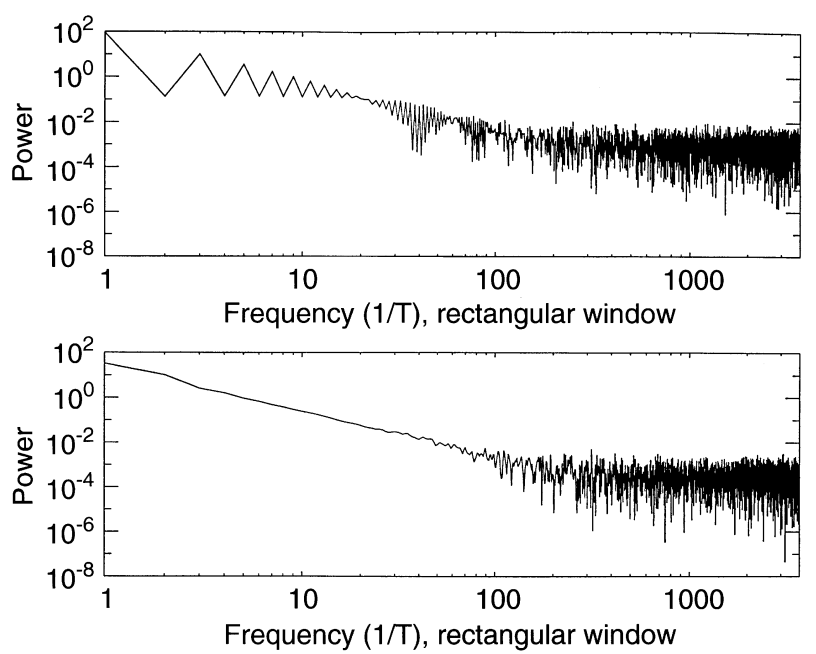

Fig. 4. The power spectrum of the time stationary, unwindowed (upper plot) and Hanning windowed (lower plot) dataset sampled by spacecraft 1 in a double logarithmic plot. The abscissa denotes the frequency in units of $\Delta f$ and the ordinate the power at the corresponding frequency. The zero frequency is omitted since it will not be used by the test 

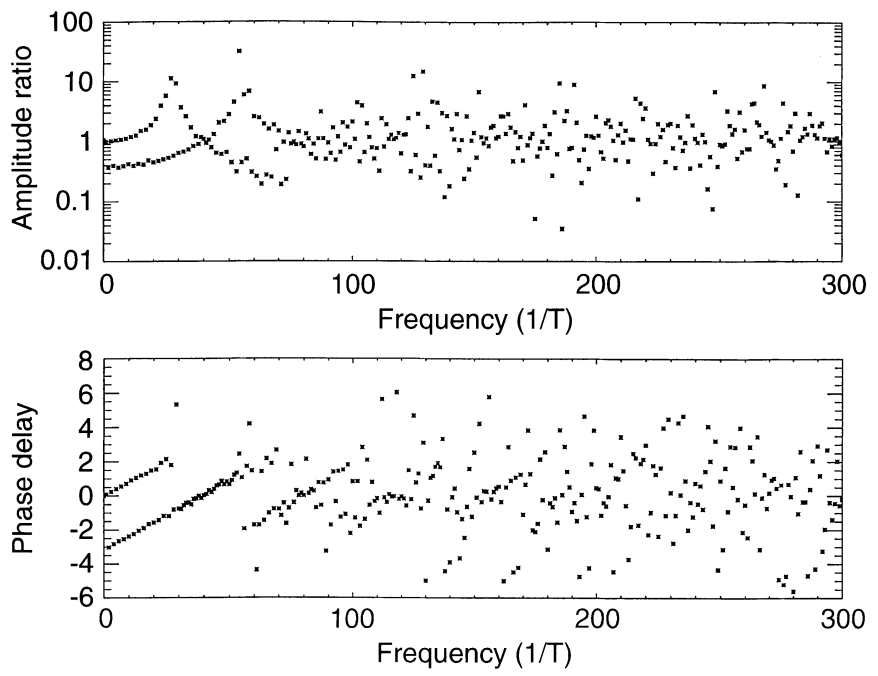

Fig. 5. The amplitude ratio/phase difference of the time stationary, unwindowed datasets. The abscissa denotes the frequency in units of $\Delta f$, the ordinate the amplitude ratio/phase difference. The zero frequency is omitted. Frequencies higher than $300 \Delta f$ are not displayed since they consist only of noise

frequency whereas the ordinate shows the amplitude ratio/phase difference between the two datasets. Even though the phase test (Eq. 2, lower plot) shows a linear phase relation between the data of both spacecraft for frequencies up to $50 \Delta f$, it yields an incorrect gradient as we shall see later. The amplitude test (Eq. 1, upper plot) is not fulfilled for any frequency range. Instead of showing a ratio of one, as would be excepted from the sampled stationary structure, the amplitude ratio jumps between higher and lower values than one. This jumping up and down is, as we will see by comparison with the Hanning windowed test's result, an artifact introduced by the non periodicity of the datasets.

By using a Hanning window in time domain, these aliasing artifacts are largely removed at low frequencies. The stationarity test's results, shown by Fig. 6, are now consistent with a stationary structure. Again, the frequency is plotted versus the amplitude ratio/phase difference. The amplitude test (1) (upper plot) is fulfilled for frequencies up to $50 \Delta f$ and approximately fulfilled for frequencies up to $150 \Delta f$. A gradual change from the strong signal region to the noise region can be seen. The fluctuations for frequencies between $50 \Delta f$ and $80 \Delta f$ are probably due to aliasing, (the signal, as can be seen from Fig. 4, is approximately one order of magnitude stronger than the noise for this range and should thus still be dominant). The Hanning window cannot remove aliasing, it can only reduce its effects for frequencies sufficiently far away from the dominant ones. Since the peak of the signal is around four orders of magnitude stronger than the signal in the frequency range between 50 and $80 \Delta f$, this might be a valid assumption.

The phase test (2) shows the same smooth change from the signal region to the noise region as the amplitude test. The fact that the stationarity test using Hanning windows yields correct results, can be verified by calculating the time delay, which is known to be
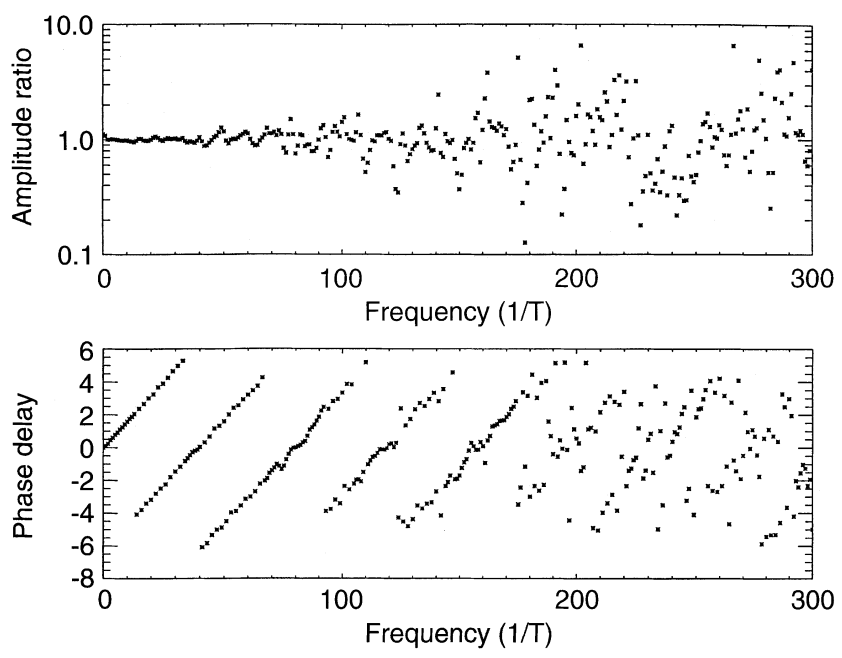

Fig. 6. The amplitude ratio and the phase difference of the time stationary, Hanning windowed datasets. The abscissas denote the frequency in units of $\Delta f$, the ordinate of the upper plot the amplitude ratio and of the lower plot the phase difference. The zero frequency as well as frequencies higher than $300 \Delta f$ are omitted

$200 \Delta x$ from Fig. 3, from the phase difference diagram. Since the spacecraft speed is $\Delta x / \Delta t$, a spatial separation of $200 \Delta x$ between the two sampling devices corresponds to a time lag in the time series of $200 \Delta t$.

From Eq. (2) one obtains by defining $\Phi_{1}(f)-\Phi_{2}(f)$ $=\Delta \Phi(f)$ :

$\delta t=\frac{\Delta \Phi(f)}{2 \pi f}$.

We will now calculate the time delays given by the phase test for the unwindowed and for the Hanning windowed datasets.

Fitting a straight line to the first 20 points of the unwindowed phase difference plot Fig. 5, where the points are shifted when necessary by $\pi$ on the ordinate, gives: $\Delta \Phi(f) / T f=0.079 \pm 0.001$. The equivalent time delay is then:

$\delta t=\frac{\Delta \Phi(f)}{2 \pi f}=(99 \pm 1) \Delta t$

where $T$ is the total sampling time of $7900 \Delta t$. This result is half the actual time lag.

The same procedure, in this case shifting by $2 \pi$, for the Hanning windowed phase difference plot Fig. 6 yields a gradient of: $\Delta \Phi(f) / T f=0.16 \pm 0.001$ which is equivalent to a time delay of:

$\delta t=\frac{\Delta \Phi(f)}{2 \pi f}=(201 \pm 1) \Delta t$

which matches the real time lag of 200 time steps within error margins. Hanning windowing the data is therefore essential for both, the amplitude and the phase test.

We can use the stationarity test (Figs. 5 and 6) to define the filter to remove nonstationary frequencies which, in the time stationary simulation, are due to noise alone. A rectangular low pass filter with $f \leq 100 \cdot \Delta f$ was multiplied in the Fourier domain to the datasets. Figure 7 shows, for the time interval $\tilde{t}=23400$ to 


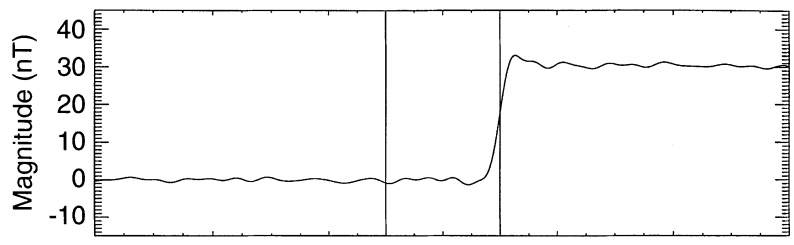

$2.34 \times 10^{4} 2.36 \times 10^{4} 2.38 \times 10^{4} 2.40 \times 10^{4} 2.42 \times 10^{4} 2.44 \times 10^{4} 2.46 \times 10^{4}$ Simulation time, Spacecraft 1

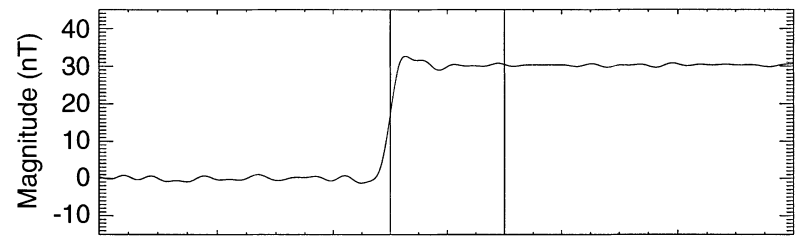

$2.34 \times 10^{4} 2.36 \times 10^{4} 2.38 \times 10^{4} 2.40 \times 10^{4} 2.42 \times 10^{4} 2.44 \times 10^{4} 2.46 \times 10^{4}$ Simulation time, Spacecraft 2

Fig. 7. The low pass filtered, time stationary datasets. The filter cut off frequencies above the threshold of $100 \Delta f$. The abscissa denotes the sampling time step $\tilde{t}$ and the ordinate the smoothed $B_{\perp}$ field magnitude

$\tilde{t}=24600$, the result of the noise removal, confirming that the structure was indeed time stationary.

\subsection{Simulation data, time evolving boundary}

The same analysis is now applied to the time evolving structure. At the time $\tilde{t}=23700$, a magnetic field parallel to the simulation direction was switched on. Comparing Fig. 1 and Fig. 2 we might expect the boundary motion in this frame to be small in the time evolving run. The stationarity test should thus extract a similar bulk motion of the boundary as in the stationary run.

Figure 8 shows the magnetic field as sampled by the two spacecraft. The upper plot corresponds to spacecraft 1, the lower one to spacecraft 2 . Shown are the samples for the time $\tilde{t}=23400$ to $\tilde{t}=24600$. They contain noise and propagating waves at the two boundaries between the two plasmas (box 1 and box $2)$. The waves generated at the boundary located at $\tilde{x} \approx 8192$ (see Fig. 2) did not travel far enough to reach a position with a sufficiently high weight of the Hanning window to contribute to the stationarity test.

The phase and amplitude test shown by Fig. 9 indicates a stationary structure for frequencies up to 40 $\Delta f$. Above that threshold and up to $140 \Delta f$, the tests imply a non stationary structure that is evolving in its rest frame which can still be distinguished from noise. The gradient of a straight line fitted to the phase difference for the first 20 points yields a time delay of $\delta t=(204 \pm 2) \Delta t$. Since the time stationary lag of 200 lies outside of the error margin, the boundary must have moved as a whole in the simulation frame.

The upper plot of Fig. 10 shows the low pass filtered and stationary components of both datasets. The abscissa denotes $\tilde{t}$. The signal was filtered in frequency space using a rectangular window with a cut off frequency of $40 \Delta f$. The structure, as seen by spacecraft
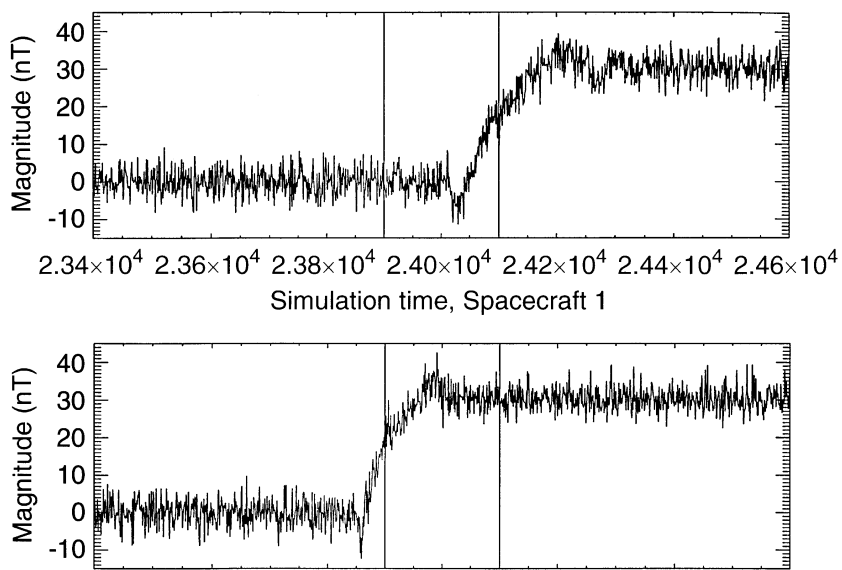

$2.34 \times 10^{4} 2.36 \times 10^{4} 2.38 \times 10^{4} 2.40 \times 10^{4} 2.42 \times 10^{4} 2.44 \times 10^{4} 2.46 \times 10^{4}$ Simulation time, Spacecraft 2

Fig. 8. The magnetic $B_{\perp}$ field component as sampled by the spacecraft. The abscissa denotes the simulation time $\tilde{t}$ and the ordinate the corresponding magnetic field for the time evolving run. The samples of higher and lower simulation time are clipped, since they only represent noise

1 (dash-dotted line) almost matches that seen by spacecraft 2 (solid line). The lower plot shows the time evolving component ( $\mathrm{f}=41$ to $140 \Delta f$ ) of the signal. The abscissa shows $\tilde{t}$. Although this bandwidth includes some of the quasi-stationary component, the signal sampled by spacecraft 1 compared with that sampled by spacecraft 2 shows, that a wave is propagating away from the boundary. After the boundary crossing, spacecraft 1 sampled considerable wave activity which does not show up in the dataset sampled by spacecraft 2 .

\section{Discussion}

The stationarity test, proposed by Chapman and Dunlop (1993) has for the first time been investigated
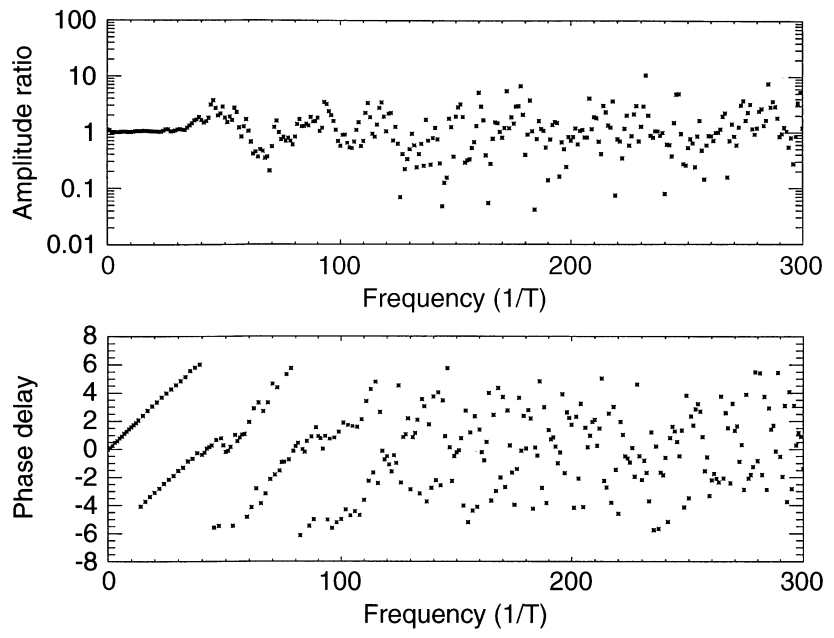

Fig. 9. The amplitude ratio and the phase difference of the time evolving, Hanning windowed datasets. The abscissas denote the frequency in units of $\Delta f$, the ordinate of the upper plot the amplitude ratio and of the lower plot the phase difference. The zero frequency and frequencies higher than $300 \Delta f$ are omitted 

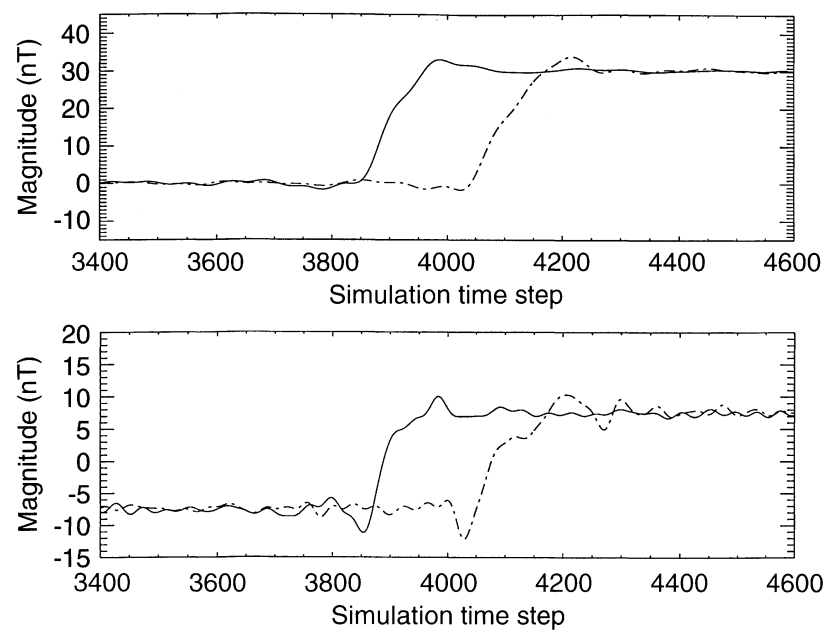

Fig. 10. The upper plot shows the low pass filtered time stationary component of the structure. The filter cut off frequencies higher than $35 \Delta f$. The abscissa corresponds to the simulation time step $\tilde{t}$. The dash dotted line denotes the magnetic $B_{\perp}$ component sampled by spacecraft 1 , the solid line $B_{\perp}$ sampled by spacecraft 2 . The lower plot shows the band pass filtered (frequencies between 41-140 $\Delta f$ are kept) time evolving component of the structure. Again, the dash dotted line corresponds to spacecraft 1

by applying it to two point measurements provided by an electromagnetic particle in cell code. By comparing the test's results for unwindowed and Hanning windowed time domain datasets, it was shown that careful windowing is crucial in order to reduce aliasing effects which would otherwise render the test meaningless. The Hanning window was chosen due to its simplicity. Other windows Hamming (1993) may yield a better performance for particular datasets but, since we are interested in a standard test, the Hanning window is a reasonably good choice.

By applying the test to two point measurements of a time stationary boundary layer with both datasets having a known time delay, the stationarity test was shown to yield correct results, within error margins for the time lag. Filtering the datasets in the frequency domain using the results of the test allowed it to remove the noise and to restore the stationary component of the datasets.

The two point measurements of the time evolving run showed that the test is also capable of extracting non stationary components like waves. In principle it might be possible to extract, from the phase difference diagram and the relative velocity of the spacecraft with respect to the plasma, the phase velocity of the wave in the plasma's rest frame. This information could, in turn, be used to determine, for example, the wavemode. If the wavemode could be detected from polarisation and field measurements (and thus the wave's phase velocity in the plasma frame of reference) the relative velocity of the spacecraft could be determined with respect to the plasma. (Both cases are possible only if the linear dispersion relation is known and applicable). When doing this, one has to consider that other structures and events represented on the considered frequency interval will mix their phase information to that of the wave, thus any such attempt has to be done cautiously.

Acknowledgements. Mark Erick Dieckmann was partially funded by a Warwick University and a CNRS Orléans studentship.

Topical Editor K.-H. Glassmeier thanks two referees for their help in evaluating this paper.

\section{References}

Chapman, S. C., and M. W. Dunlop, Some consequences of the shift theorem for multispacecraft measurements, Geophys. Res. Lett., 20, 2023-2026, 1993.

Cluster Workshops, ESA, SP-371, Braunschweig 28-30 September 1994 and Toulouse 16-17 November 1994.

Devine, P. E., S. C. Chapman, and J. W. Eastwood, One- and twodimensional simulations of whistler mode waves in an anisotropic plasma, J. Geophys. Res., 100, 17 189-17 203, 1995.

Dunlop, M. W., Review of the Cluster orbit and separation strategy: consequence for measurements, ESA SP-306, 1990.

Dunlop, M. W., D. J. Southwood, K. H. Glassmeier, and F. M. Neubauer, Analysis of multipoint magnetometer data, $A d v$. Space Res., 8, 273-277, 1988.

Dunlop, M. W., A. Balogh, D. J. Southwood, R. C. Elphic, K. H. Glassmeier, and F. M. Neubauer, Configurational sensitivity of multipoint magnetic field measurements, ESA SP-306, 1990.

Hamming, R. W., Digital filters, ISBN 0132128128, Prentice Hall, 1993.

Neubauer, F. M., K. H. Glassmeier, R. Walter, and M. W. Dunlop, Cluster as a wave telescope, ESA SP-306, 1990. 